\title{
Editorial
}

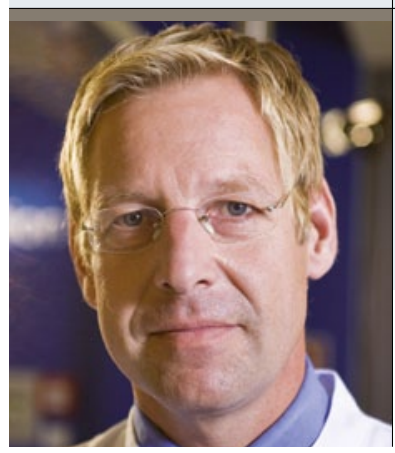

"Die Generation Y wird die Zukunft einer hervorragenden

Patientenversorgung in Deutschland sichern - sofern Kostenträger

und Politik erkennen, dass Gesundheit keine Last, sondern ein un-

angreifbar sinnvolles Ziel für Ausgaben und Investitionen darstellt."

Prof. Dr. med. Christian Gerloff, Direktor der Klinik und Poliklinik für Neurologie, Universitätsklinikum Hamburg-Eppendorf

\section{Generation Y}

U nlängst hat die Frankfurter Allgemeine Zeitung (FAZ) über die „Millennials“, auch „Generation Y“" genannt, in der Medizin geschrieben: Sie sind zwischen 1980 und 1990 geboren, überwiegend gut ausgebildet und stellen die erste Generation dar, die in einer Umgebung von Internet und mobiler Kommunikation aufgewachsen ist. Man nennt sie daher auch „Digital Natives“. Im Artikel vom 27. April 2012 schreibt die FAZ über die Generation Y: „Sie lehnen Hierarchien ab und wollen lieber geregelte Arbeitszeiten als steile Karrieren: Die nach 1980 geborenen Ärzte werden zur Herausforderung im deutschen Klinikalltag."

Moment mal ... Verkehrte Welt?

Wie nannte man noch die Generation, die jetzt in der Medizin über etwas mehr Erfahrung verfügt und an vielen Stellen das Steuer in der Hand hat? Richtig: „Generation X“ ... Gerade einmal einen Buchstaben und ein paar Jahre entfernt, definiert als die in den 1960er- und 1970er-Jahren geborene Generation. Diese zeichnet sich, so sagt man, dadurch aus, dass sie erstmals erleben musste, wie ohne Krieg oder andere Katastrophen Wohlstand und ökonomische Sicherheit in der Gesellschaft abnahmen. Das schaffte Druck. Die Generation X habe ein neues Wertesystem erkannt und in diesem Kontext wurde ironisch auch von „exhibitionistischer Bescheidenheit“ gesprochen.

\section{Exhibitionistische Bescheidenheit?}

Absolventen des Studiums für Humanmedizin aus der Generation X mussten sich zu Dutzenden auf einzelne AiP-(für die Jüngeren: Arzt im Praktikum-) Stellen bewerben. Absagen jagten Absagen und dann endlich der Triumph: ein 18-Monatsvertrag mit einer Bruttovergütung von rund $1.000 €$ pro Monat. Diese $1.000 €$ Monatsgehalt beziehen sich auf die Zeit kurz vor der Abschaffung des AiP, also 2004. Davor waren die Gehälter noch niedriger. War das „exhibitionistische Bescheidenheit"? Fehlanzeige.
Und noch mehr: Zurückkommend auf den FAZArtikel müssten wir nun ableiten, dass die Generation X Hierarchien gut fand und steile Karrieren den geregelten Arbeitszeiten vorzog. Außerdem war die Generation X - im Gegensatz zu Y - keine „Herausforderung im deutschen Klinikalltag". Das hat in der Tat den schalen Beigeschmack von „früher war alles anders und insbesondere besser". Romantische Verklärung und nochmals Fehlanzeige.

Meine These: Die Ärztinnen und Ärzte der Generation $\mathrm{X}$ waren und sind nicht besser, menschlicher, empathischer oder teamfähiger als diejenigen der Generation Y. Ich sehe auch keine jungen Menschen in der Weiterbildung für Neurologie, die lieber in „virtuellen Netzen“ als in einem waschechten Team arbeiten. Vielleicht arbeiten sie sogar besser im Team als die Generation X. Denn die zunehmende Komplexität der Medizin, die digitale und mobile Verfügbarkeit von Informationen im Sekundentakt machen ein hochgradig vernetztes Denken und Arbeiten möglich und nötig.

\section{Neurowissenschaften boomen}

Warum entsteht dann dieser Eindruck? Ich wiederhole: „Die nach 1980 geborenen Ärzte werden zur Herausforderung im deutschen Klinikalltag“ (FAZ). Sicherlich hat sich generell die Kommunikationskultur geändert, sicherlich sind die Hierarchien flacher geworden - nicht nur in der Medizin. Hand aufs Herz, auch die Generation X hat für geregelte Arbeitszeiten gekämpft, hat für die Abschaffung des AiP gekämpft (mit Erfolg) und hatte wenig Freude an intransparenten Hierarchien. Was hat sich also geändert? Die Medizin ist noch spannender geworden, die Neurologie im Besonderen hat riesige Schritte nach vorne und in Richtung einer modernen therapeutisch orientierten Disziplin gemacht. Die Neurowissenschaften boomen, die Herausforderungen steigen. Gleichzeitig gelingt es uns Medizinern nicht, die Rahmenbedingungen 


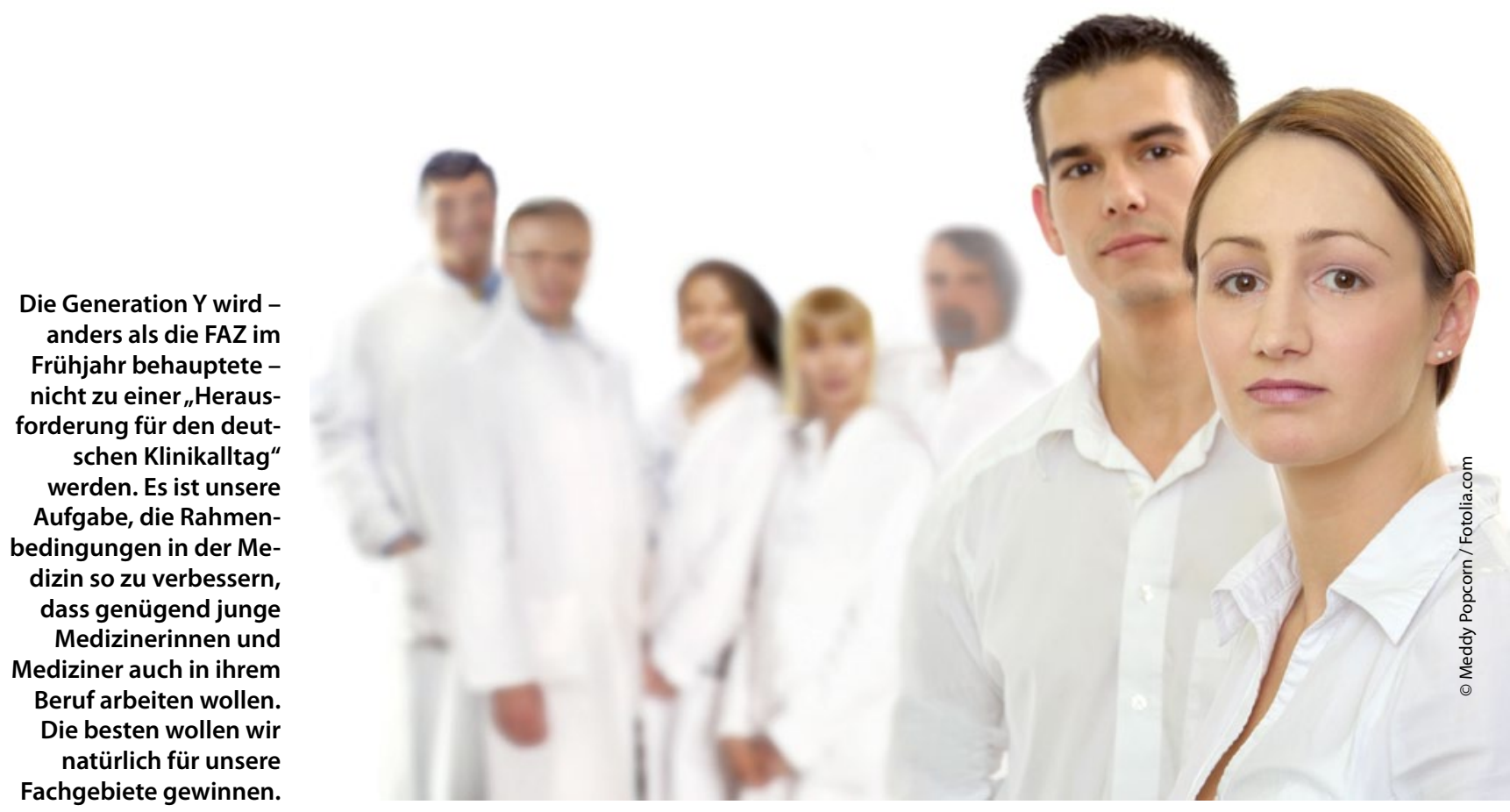

so zu gestalten, dass wir im "Steady-State“ mit angemessenem ökonomischem Druck arbeiten. Einen angemessenen Druck hält jeder aus, auch wir haben kein Interesse, Ressourcen zu verschwenden. Hier aber krankt das System. Geld bekommen wir nur von einer, maximal von zwei Instanzen: Kostenträger und Staat. Die einen machen derzeit deftige Überschüsse, geben das aber nicht an uns weiter. Die anderen sind in der „Krise“, weil einige sich verzockt haben. Also sind auch die Staatskassen leer. Duale Finanzierung versus Investitionsstau. Hier liegt doch der Hase im Pfeffer. Je weiter man in der ärztlichen „Hierarchie“ kommt, desto mehr beschäftigt man sich mit den Themen „Ökonomisierung“, „Prozessoptimierung“, „CMI-Steigerung“, „Deckungsbeitragsrechnung“, zugleich „Kundenorientierung“, „Beschwerdemanagement“, „Belegungsmanagement“, „Entlassmanagement" und so weiter und so fort. Die Liste ist lang. Ärztliches Handeln - dafür muss man sich schon aktiv Zeit erkämpfen. Vielleicht sollte man einmal darüber nachdenken, ob diese Aspekte nicht auch ein Grund dafür sind, warum die „steile Karriere“ zu Oberarzt und Chefarzt (respektive -ärztin) nicht mehr ganz so attraktiv ist. Und es ist selbst dem romantischsten Weiterbildungsassistenten klar, dass leitende Positionen in der Medizin heute nicht mehr viel mit jenen vor 20 Jahren zu tun haben.

\section{Rahmenbedingungen verbessern}

Ich wage die Hypothese, dass die Generation Y heute genauso begabt, gut ausgebildet und engagiert ist wie die Generation X oder jede beliebige andere Generation in der Medizin. Statt hier eine „Evolution“ ärztlicher Grundeinstellungen in so kurzen Zeitfenstern wie 20 oder 30
Jahren zu sehen, sollten wir lieber daran arbeiten, die Rahmenbedingungen für unser Schaffen zu verbessern.

Die nach 1980 geborenen Ärzte werden nicht „zur Herausforderung im deutschen Klinikalltag“, sondern sie sind diejenigen, die die Zukunft einer hervorragenden Patientenversorgung in Deutschland sichern - sofern Kostenträger und Politik erkennen, dass Gesundheit keine Last, sondern ein unangreifbar sinnvolles Ziel für Ausgaben und Investitionen darstellt. In dieser Hinsicht sind sich definitiv Generation X und Y einig.

\section{DGN-Kongress in Hamburg}

Die Nachwuchsförderung hat sich die Deutsche Gesellschaft für Neurologie (DGN) seit Jahren auf die Fahnen geschrieben. Auch auf der kommenden Tagung in Hamburg wird es eigens ein Programm der Jungen Neurologen geben. Die Veranstaltungen finden im „DGN(forum“ in Halle 3 statt. Kongressprogramm und Fortbildungsakademie können sich auch in diesem Jahr in jeder Hinsicht sehen lassen. Ganz besonders aufmerksam machen möchte ich Sie auf das Präsidentensymposium zum Thema „Grenzen und Ziele der Neurologie 2012“ am Donnerstag, den 27. September ab 15.00 Uhr. Details finden Sie unter: www.dgnkongress.org.

Ich freue mich, Sie in Hamburg zu sehen!

Ihr

Christian Gerloff

Kongresspräsident des 85. DGN-Kongresses 